\title{
Transformative Lernangebote kritisch-reflexiv gestalten. Fachdidaktische Orientierungen einer emanzipatorischen BNE
}

\author{
fabian.pettig@uni-graz.at, Institut für Geographie und Raumforschung, Universität Graz
}

eingereicht am: 09.12.2020, akzeptiert am: 19.02.2021

Der Beitrag fragt nach den Gelingensbedingungen transformativer Lernprozesse im Geographie- und Wirtschaftskundeunterricht (GW-Unterricht). Ausgehend von der Kritik an der instrumentellen Auslegung einer Bildung für Nachhaltige Entwicklung (BNE) wird das Potenzial transformativen Lernens für eine emanzipatorische BNE ausgelotet. Unter Rückgriff auf einen pädagogischen Lernbegriff mündet dieses Vorgehen in die Konzeptualisierung eines Handlungsrahmens zur Gestaltung eines kritisch-reflexiven GW-Unterrichts im Horizont gesellschaftlicher Transformationsprozesse.

Keywords: Transformatives Lernen, Bildung für nachhaltige Entwicklung, Degrowth, Bildungstheorie, Umlernen

\section{Designing transformative learning opportunities critically and reflexive. Didactical orientations of an emancipatory ESD}

\begin{abstract}
This article examines the conditions for the success of transformative learning in geography and socioeconomic education. Starting from the criticism of instrumental ESD-approaches, the potential of transformative learning for emancipatory ESD is explored. With recourse to a pedagogical concept of learning, this leads to the conceptualization of a framework for designing critical and reflexive geography and economics lessons in the horizon of social transformation.
\end{abstract}

Keywords: transformative learning, education for sustainable development, degrowth, education theory, relearning

\section{$1 \quad$ Einleitung}

Die deutschsprachige Nachhaltigkeitsdebatte wird seit dem WBGU-Gutachten Welt im Wandel vom Begriff der „Großen Transformation“ (WBGU 2011) geprägt. Diese „beschreibt einen massiven ökologischen, technologischen, ökonomischen, institutionellen und kulturellen Umbruchprozess zu Beginn des 21. Jahrhunderts" (Schneidewind 2019: 11). Ziel dieses Prozesses ist es „gleiche und hinreichende Entfaltungschancen für die Menschen auf diesem Planeten, heute und zukünftig - innerhalb der bestehenden planetaren Grenzen“" (ebd.) zu ermöglichen. Dabei zeichnet sich die vielstimmige Diskussion zur konkreten Umsetzung solch eines Transformationsprozesses durch konkurrierende Meinungen und strittige Positionen aus, welche Visionen unterschiedlicher Zukünfte entwerfen, die teilweise unvereinbar sind. So reichen aktuell wissenschaftlich, politisch wie gesellschaftlich verhandelte Ideen zu einem zukunftsfähigen post-fossilen Wirt- schaftssystem von neoökologischen Ansätzen i.S.v. Green Growth hin zu alternativen Ökonomien i.S.v. Degrowth. Angesichts globaler Umweltkrisen, dem 1,5-Grad-Ziel und der hiermit verbundenen Rolle und Verantwortung des Menschen im Zeitalter des „Anthropozäns" (Crutzen \& Stoermer 2000; Crutzen 2002), scheint die Notwendigkeit eines umfassenden Umdenkens zwar wissenschaftlich weitgehend unumstritten, die Art und Weise, wie ein zukunftsfähiges Miteinander aussehen kann bzw. muss, zugleich jedoch auf unterschiedlichen Ebenen höchst umstritten zu sein.

Für Bildungskontexte liegen in dieser komplexen, unsicheren und auf ungewisse Zukünfte hin ausgerichteten Wissensbasis mehrere grundsätzliche Herausforderungen begründet. So muss es im Fachunterricht Schüler*innen sowohl ermöglicht werden, ein Problembewusstsein angesichts planetarer Grenzen und der Unumkehrbarkeit bestimmter erdsystemarer Entwicklungen - u. a. Biologische Vielfalt, Klimawandel, Globale Stoffkreisläufe (vgl. Steffen et al. 2015) - 
auszubilden, als auch sich begründet zu kontroversen Strategien von Nachhaltigkeit bzw. nachhaltiger Entwicklung zu positionieren, eigene Haltungen zu diesen einzunehmen und sich an der Gestaltung einer zukunftsfähigen Welt und Gesellschaft aktiv beteiligen zu können. Mit Bezug hierauf wird in der deutschsprachigen BNE-Debatte das - ebenfalls im bereits erwähnten WBGU-Gutachten eingeführte - Konzept "Transformativer Bildung" (WBGU 2011) diskutiert. Dieses soll ein „Verständnis für Handlungsoptionen und Lösungsansätze“, bspw. über „Innovationen, von denen eine transformative Wirkung zu erwarten oder bereits eingetreten ist" generieren (ebd.: 25). Bildungseinrichtungen käme laut WBGU dabei die Aufgabe zu „nachhaltigkeitsorientiertes Wissen“ (ebd.), u. a. zu Mobilität, Ernährung und generationenübergreifender Verantwortung (ebd.: 374) zu vermitteln. Dahinter steht das Ziel „dem Handeln Einzelner die notwendige Richtung“ zu geben (ebd.). Diese Zielsetzung wird in der jüngst erschienenen BNE-Roadmap 2030 im Rahmen des UNESCO-Programms Education for Sustainable Development: Towards achieving the $S D G s$ noch schärfer formuliert: "How can we encourage learners to undertake transformative actions for sustainability to shape a different future, before it is too late?" (UNESCO 2020: 9).

Für die konkrete Unterrichtspraxis wird hierin jedoch ein bildungstheoretisches Problem evident, welches die BNE-Debatte insgesamt kennzeichnet: „Es stellt sich immer wieder neu die Frage, wo (legitime) Erziehungsbemühungen in (illegitime, wenn auch vielleicht unbeabsichtigte) Indoktrinationsversuche umschlagen" (Marchand 2015: 141). Denn kritische Bildung ist zuallererst ein Prozess der Emanzipation, d.h. der Förderung des eigenen Denkens und Handelns anhand der Reflexion und Dekonstruktion herrschender Machtverhältnisse und Narrative, um mündig für die eigenen Überzeugungen einstehen und diese über Teilhabe an demokratischen Aushandlungsprozessen verwirklichen zu können. Mündigkeit beschreibt also sowohl die Fähigkeit zur Selbstbestimmung als auch zur Urteilsfähigkeit angesichts komplexer und kontroverser Problemlagen in einem politisch bildenden Fachunterricht. Aufgrund der vielfältigen, teils widersprüchlichen, Verwendungskontexte des Begriffs Transformativer Bildung „läuft er [jedoch] Gefahr unter dem Deckmantel emanzipatorischer Anliegen ein Instrument des Change Managements neoliberaler Umstrukturierungen zu werden" (Lingenfelder 2020: 55).

Der Beitrag verfolgt vor diesem Problemhintergrund zwei miteinander zusammenhängende Ziele: Erstens soll unter Rückgriff auf einen pädagogischen Lernbegriff ein Beitrag zur bildungstheoretischen Fundierung und damit zur konzeptionellen Klärung
Transformativer Bildung geleistet werden; zweitens soll diese Begriffsreflexion in die Konzeptualisierung eines Handlungsrahmens zur Gestaltung transformativer Lernprozesse und Bildungsangebote in einem kritisch-reflexiven GW-Unterricht münden.

\section{Das normative Konzept BNE in Schule und Unterricht}

Die Vereinten Nationen riefen die BNE-Dekade (2005-2014) mit dem Ziel aus, "to integrate the principles, values, and practices of sustainable development into all aspects of education and learning" (UNESCO 2005: 6). Für Deutschland liegt inzwischen eine umfassende Längsschnittstudie ${ }^{1}$ vor, welche u. a. die curriculare Implementierung einer BNE in das deutsche Schulsystem analysiert (Holst et al. 2020). Hier zeigt sich, dass BNE zwar in allen untersuchten Bildungsplänen ${ }^{2}$ Einzug gehalten hat, deren quantitative und qualitative Implementierung aber deutliche Unterschiede aufweist; insbesondere die in jüngerer Zeit überarbeiteten Bildungspläne weisen eine breite und umfassende Implementierung einer BNE auf (ebd.: 8). Während BNE in manchen Bildungsplänen als transdisziplinäres Anliegen verfolgt wird, kommt sie in anderen Plänen hauptsächlich in Fächern mit hoher Affinität zur Nachhaltigkeit, bspw. der Geographie, vor (ebd.). Zwar liegt für Österreich keine vergleichbar umfassende Erfassung der curricularen Implementierung einer BNE vor, jedoch wurde die Aufforderung der Vereinten $\mathrm{Na}$ tionen im Kontext der „Österreichische[n] Strategie zur Bildung für nachhaltige Entwicklung" (BMWF 2008) aufgegriffen und u. a. auch per „Grundsatzerlass Umweltbildung für nachhaltige Entwicklung" (BMBF 2014) für alle Schulstufen und Schularten als wesentlicher Bezugspunkt der österreichischen Bildungslandschaft definiert. Im aktuellen Lehrplanentwurf für das Schulfach GW vom August 2020 wird weiters überdeutlich, dass BNE bzw. Nachhaltigkeit und nachhaltige Entwicklung wesentliche Bezugspunkte in der Konkretisierung der Lehr- und Bildungsaufgabe, den didaktischen Grundsätzen, den ausgewiesenen Kompetenzbereichen sowie den Basiskonzepten des Faches darstellen. ${ }^{3}$

\footnotetext{
Erste Erhebung 03/2017, zweite Erhebung 10/2019 (Holst et al. 2020: 3)

2 Im Rahmen der Studie wurden insgesamt 316 Bildungspläne von neun ausgewählten Fächern (Deutsch, Englisch, Wirtschaft, Politik, Ethik/Philosophie, Geographie, Chemie, Biologie, Informatik) aller 16 Bundesländer für die Klassenstufen 4 und 9 (aller Schularten) untersucht (Holst et al. 2020: 4).

3 Die Veröffentlichung des überarbeiteten Lehrplans ist für Herbst 2021 geplant; der neue Lehrplan soll mit dem Schuljahr 2023/24 im Schulsystem wirksam werden. Die Abgabeversion
} 
Die bildungspolitisch motivierte Implementierung von BNE wird von Beginn an auch äußerst kritisch diskutiert. Hasse (2006: 40) weist bereits früh in der geographiedidaktischen Diskussion darauf hin, dass die Entwicklung von der Umweltbildung zur Bildung für nachhaltige Entwicklung keine paradigmatische Neuausrichtung darstellt, sondern eine Reihe an bereits seit Längerem diskutierten Problemen schlicht unter neuen Begrifflichkeiten reproduziert und dabei die geführten Diskussionen ausblendet. So liegt bspw. beiden Ansätzen ein Verständnis von Bildung zugrunde, welches auf die zweckgebundene Vermittlung von Wissen abzielt, um auf diese Weise auf die Handlungen der Lernenden einzuwirken. Damit verbunden wird grundsätzlich kritisiert, dass es sich bei BNE eher um ein normativpolitisches, denn bildungstheoretisch motiviertes Konzept handelt (Schuler \& Kanwischer 2013). Im Besonderen werden Verschleierungstendenzen in BNE-Konzepten hervorgehoben (Budke \& Gryl 2016). So werden einerseits Interessenkonflikte und Machtasymmetrien kaum beachtet, woraus die Gefahr entsteht, einfache Lösungen globaler Probleme zu vermitteln, statt multiperspektivisches Verstehen und Bewusstsein für deren komplexe Verflechtungen anzubahnen. Andererseits wird die Vereinbarung der Nachhaltigkeitsdimensionen als utopisches Machbarkeitsideal formuliert und deren Erfüllung dem Individuum auferlegt. D.h., dass die Verantwortung für die nachhaltige Transformation vornehmlich dem*der Einzelnen zugeschrieben wird, wenn bspw. lediglich die individuelle Mobilität oder der individuelle Konsum zugunsten nachhaltiger Entwicklung geändert werden sollen (vgl. Danielzik 2013). Hierin zeigt sich eine immanente Widersprüchlichkeit nachhaltiger Entwicklung: „Während sie die Individuen auf Suffizienz einstimmt, bleiben die Systeme industrialisierter Gesellschaften auf $\mathrm{Zu}$ wachs eingestellt" (Kaufmann 2004: 180). Diese neoliberale Tendenz einer "kompensatorische[n] Eigenverantwortung" (Lehner \& Gryl 2019: 12-13) geht dabei mit der Verschleierung systemimmanenter nicht-nachhaltiger Strukturen einher. Denn die Fokussierung der Symptomebene vernachlässigt das Verstehen komplexer Hintergründe und suggeriert einfache Lösungen und individuelle Pflichten, ohne die „Prämissen neoliberaler Wachstumsideologie als Ursache [...] grundsätzlich infrage zu stellen" (Kehren \& Winkler 2019: 379). Die BNE würde damit ins Leere laufen, wenn etwas vermittelt werden soll, „was es noch nicht gibt und angesichts des Festhal-

des Entwurfs an das Bildungsministerium ist online einsehbar: https://www.eduacademy.at/gwb/pluginfile.php/39161/ mod_resource/content/3/gw_Lehrplan_\%0Bfuenftversion_ Aug2020_layoutiert.pdf (06.12.2020) tens an den etablierten Produktionsverhältnissen so auch nicht geben kann" (ebd.: 377).

Diese Kritikpunkte spiegeln sich letztlich auch in den „17 Sustainable Development Goals“ (UN 2015) wider, in denen u.a. zwar „eine Forcierung von Reflexivität eingefordert [wird] und sich das Programm von neoliberalen Bildungskonzepten abzugrenzen versucht" (Scharf \& Gryl 2020: 28), dieses aber „lediglich im vorgegebenen Rahmen" (ebd.) abläuft, das Bildungskonzept selbst und deren eingeschriebenen Logiken also nicht explizit Gegenstand der Diskussion sind. Auch in der internationalen Debatte wird pointiert zusammengefasst, dass die zunehmende Verankerung von BNE in Institutionen und (Bildungs-) Systemen eher durch ein neoliberal-pragmatisches denn kritisch-problematisierendes Verständnis von Bildung und Nachhaltigkeit gekennzeichnet ist (Selby \& Kagawa 2010: 39): Internationale nachhaltigkeitsbezogene Agenden würden demnach in der Breite implizit (1) ökonomisches Wachstum als Leitmotiv reproduzieren, (2) einen utilitaristischen Blick auf $\mathrm{Na}$ tur als Ressource anlegen, (3) die Technologie-Wachstums-Orientierung globaler Märkte widerspiegeln und (4) auf Standardisierung und Messbarkeit (von Implementierungs- und Lernerfolgen) fokussieren (ebd.: 40, eig. Übersetzung).

Die Thematisierung nachhaltigkeitsbezogener Inhalte in einem politisch bildenden GW-Unterricht ist zwangsläufig in dem hier skizzierten, der BNE inhärenten Spannungsfeld situiert, welches sich zwischen der Umsetzung einer bildungspolitischen Agenda einerseits und der Ermöglichung emanzipatorischer und reflexiver Bildungsprozesse andererseits aufspannt. In der internationalen Debatte hat sich vor diesem Hintergrund die Unterscheidung in zwei aufeinander bezogene Dimensionen einer BNE bereits vor Längerem etabliert:

"We see ESD 1 as the promotion of informed, skilled behaviours and ways of thinking, useful in the short-term where the need for this is clearly identified and agreed, and ESD 2 as building capacity to think critically about what experts say and to test ideas, exploring the dilemmas and contradictions inherent in sustainable living." (Vare \& Scott 2007: 191)

Ähnlich argumentieren auch Wals et al. (2008), die instrumentelle und emanzipatorische Ansätze in der Nachhaltigkeitsbildung voneinander unterscheiden. Bei der instrumentellen BNE 1 steht die Vermittlung von Expert*innenwissen zu Werten und Verhaltensweisen im Vordergrund, die grundsätzlich anerkannt und mit einer nachhaltigen Entwicklung verbunden sind (vgl. Vare \& Scott 2007: 192; Wals et al. 
2008: 56). Damit wird darauf abgezielt, solche Verhaltensweisen zu fördern, die als nachhaltig eingeschätzt werden, womit letztlich für eine nachhaltige Entwicklung gelernt wird. Demgegenüber befähigt die emanzipatorische BNE 2 zu einer kritischen Auseinandersetzung mit einer nachhaltigen Entwicklung sowie mit der Komplexität, der Unsicherheit und den Widersprüchen, die mit ihr verbunden sind (Vare \& Scott 2007: 193f.). Die globale Transformation braucht folglich auch "a more systemic and reflexive way of thinking and acting with the realization that our world is one of continuous change and ever-present uncertainty" (Wals et al. 2008: 57). Im Rahmen einer BNE 2 geht es somit darum, angesichts dieser Komplexität und Unsicherheit begründete und vernünftige Entscheidungen zu treffen (Vare \& Scott 2007: 134). Nachhaltige Entwicklung wird dann selbst als Lernprozess verstanden. Zwar betonen Vare \& Scott, dass beide Dimensionen einer BNE in Bildungskontexten ineinandergreifen müssen, deren Verhältnis also nicht dialektisch, sondern komplementär zu denken ist, betonen aber die Bedeutung einer BNE 2, da BNE 1 erst durch diese Bedeutung erlangte. Demgegenüber verdeutlicht die bereits erwähnte Studienlage (Wals et al. 2008; Selby \& Kagawa 2010) zur curricularen und institutionellen Implementierung einer BNE aber die dabei vorherrschende, vornehmlich einseitige Auslegung i.S. einer BNE 1. In dieser einseitigen Auslegung liegt die Gefahr, Bildung für eine politische Agenda zu instrumentalisieren und damit ihres kritisch-emanzipatorischen Anspruchs zu berauben (vgl. Euler 2014).

Dass solch eine Engführung nachhaltigkeitsbezogener Bildungsangebote im Sinne einer BNE 1 in einem politisch-bildenden, d.h. mündigkeitsorientierten, GW-Unterricht nicht vertretbar ist, liegt auf der Hand. Einerseits würde ein Unterricht, welcher Alternativen zu hegemonialen (Nachhaltigkeits-)Diskursen und ein Denken in (nachhaltigkeitsbezogenen) Widersprüchen nicht zulässt bzw. ausklammert, dem Lehranspruch eines ,emanzipatorischen Vermittlungsinteresses" (Vielhaber 1999) widersprechen. Im Unterschied zur rein zweckrationalen oder handlungsbezogenen Bearbeitung von Sachverhalten innerhalb bestimmter Normen eines gesellschaftlichen Systems, wird in einem emanzipatorischen GW-Unterricht auch der herrschende Handlungsrahmen selbst zum Gegenstand der Reflexion und nach möglichen Alternativen oder Widersprüchen gesucht, um Mündigkeit und Selbstbestimmung als reflexive Praxis zu ermöglichen (Vielhaber 1999: 17). Andererseits würde solch ein Unterricht dem „Beutelsbacher Konsens“(Wehling 1977), insbesondere dem Kontroversitätsgebot und dem Überwältigungs- bzw. Indoktrinationsverbot, zuwiderlaufen (vgl. für Österreich auch BMBF 2015).
Dementsprechend muss eine emanzipatorische BNE „die Einsicht in nicht-nachhaltige Prozesse ermöglichen und über die Reflexion der Ursachen ein Problembewusstsein eröffnen, welches vielleicht imstande ist, nachhaltige Auswege aus den globalen Krisen ins Auge zu fassen" (Kehren \& Winkler 2019:377). Hierunter fällt auch das Verstehen der „Historizität und Polizität von Erziehung, Bildung, Nachhaltigkeit und der von ihnen zu verhandelnden Gegenstände" als zentrale Bildungsaufgabe im Kontext gesellschaftlicher Transformation (ebd.: 381). Damit transformative Bildungsangebote im Sinne einer BNE 2 also emanzipatorisch sind und tatsächlich Impulse in der zuvor skizzierten instrumentellen Nachhaltigkeitsdebatte setzen, muss das Konzept der Transformativen Bildung es im Sinne eines kritisch-emanzipatorischen Bildungsverständnisses ermöglichen, die eigene Disposition $\mathrm{zu}$ nachhaltigkeitsbezogenen Fragestellungen in deren komplizierten und widersprüchlichen sozialen, ökonomischen, politischen und kulturellen Verflechtungen zu reflektieren. Die bildungstheoretische bzw. erziehungswissenschaftliche Fundierung Transformativer Bildung stellt dabei zurzeit allerdings noch ein Desiderat dar (vgl. Singer-Brodowski 2016). Im Anschluss an das aus der nordamerikanischen Erwachsenenbildung stammende Konzept transformative learning ${ }^{4}$ (Mezirow 1997) wird diese Lücke aktuell zu schließen versucht.

\section{Transformatives Lernen im Horizont einer emanzipatorischen $\mathrm{BNE}$}

Es soll hier auf das transformative Lernen sowie dessen Weiterentwicklungen in der internationalen Diskussion eingegangen werden, wie sie auch in der deutschsprachigen Debatte um BNE aktuell diskutiert wird, um dessen Potenzial für eine emanzipatorische BNE herauszustellen. In den 1980er und 1990er-Jahren begründete Jack Mezirow im Kontext der nordamerikanischen Erwachsenenbildung den Begriff des „transformativen Lernens“. Mezirow (1997) unterscheidet dabei drei aufeinander aufbauende Phasen transformativen Lernens: Dekonstruktion, bspw. durch Auseinandersetzung mit Dilemmata; Rekonstruktion, d.h. das Teilen von Erfahrungen und die gemeinsame kritische Reflexion, sodass neue Perspektiven aufgebaut und ausprobiert werden können; Integration, also die Aufnahme neuer Bedeutungsperspektiven in das eigene Leben zur konstruktiven Lösung des an-

Zur Wahrung des Leseflusses wird hier der Ansatz transformative learning als transformatives Lernen übersetzt. Im Deutschen ist diese Festlegung begrifflich nicht unproblematisch, da das transformative learning dem Konzept Bildung sehr nah steht, für das es im Englischen keinen entsprechenden Begriff gibt. 
fänglichen Dilemmas (vgl. Schild et al. 2019: 34). Transformatives Lernen beschreibt also nicht lediglich eine Erweiterung von Wissen, Fähigkeiten oder Fertigkeiten, sondern eine qualitative Veränderung von Selbst- und Weltbildern. Diese Veränderungen beziehen sich auf erlernte Muster des Denkens-, Fühlens und Handelns, gesellschaftliche Leitbilder sowie orientierende Normen und Werte. In dieser Hinsicht steht das transformative Lernen einem transformatorischen Bildungsbegriff nah, auch wenn diese in unterschiedlichen Diskursen und aus einer anderen Theorietradition heraus konzeptualisiert wurden (Koller 2017). Beide Konzepte fußen auf ähnlichen Grundannahmen, bspw. werden beide aus der Kritik an einem kumulativen Lernbegriff heraus formuliert und bestimmen krisenhafte Erlebnisse als Ausgangspunkt der beschriebenen Transformationsprozesse (Nohl 2017: 97).

In Weiterentwicklungen des transformativen Lernens wurde die Veränderung individueller Bedeutungsperspektiven um die jeweiligen gesellschaftlichen und kulturellen Rahmenbedingungen, und wie diese unsere Moralvorstellungen, Wertesysteme, Erfahrungen und Handlungen prägen, erweitert und das transformative Lernen auch explizit in den Kontext nachhaltiger Entwicklung gestellt (u. a. Brookfield 2000, 2012; Sterling 2011; O'Sullivan 1999, 2002, 2012). Transformatives Lernen zielt in diesem Kontext darauf ab, implizite Macht- und Deutungsstrukturen sowie gesellschaftliche Narrative aufzudecken, die Enkulturation unserer Sicht- und Denkweisen erfahrbar zu machen, diese in einem reflexiven Prozess verhandelbar zu machen und hierüber prinzipiell einer Transformation zugänglich zu machen. Es geht dann z. B. um den Wandel der Bedeutungsperspektiven in Bezug auf Vorstellungen von Mensch-Natur-Verhältnissen, zu gesellschaftlichen Machtverhältnissen oder zu globaler Gerechtigkeit ebenso wie um Selbstwirksamkeitserfahrungen in Bezug auf gesellschaftliche Transformationsprozesse und die Artikulation eigener Visionen des Zusammenlebens. Es sollen Menschen dazu befähigt werden, eine globale Transformation hin zu einer gerechten und zukunftsfähigen Welt demokratisch mitgestalten zu können. Im Unterschied zum Aufbau von Wissen, Fähigkeiten und Fertigkeiten zur Förderung eines ,nachhaltigen Lebensstils wird also das Erlangen von Mündigkeit im Horizont der Notwendigkeit gesellschaftlicher Transformation fokussiert.

Gelingendes transformatives Lernen muss daher im Anschluss an die Kritik an BNE für globale Verflechtungen nicht-nachhaltiger Strukturen und Machtverhältnisse sowie die Dilemmata nachhaltiger Entwicklung sensibilisieren, die eigene Eingebundenheit in diese Verhältnisse reflektieren und die Ausbildung eigener Meinungen und Haltungen ermöglichen. Neben dem hegemonialen - und die Nachhaltigkeitsdebatte dominierenden - Green Growth-Narrativ müssten folglich auch Alternative Ökonomien und Wohlstandsmodelle i.S.v. Degrowth, wie die Commons-Bewegung, Postwachstumsökonomien oder Gemeinwohlökonomie (vgl. Getzin \& Singer-Brodowski 2016: 36f.), stärkere Sichtbarkeit in Bildungskontexten erlangen. Es darf dabei die Kritik hegemonialer Narrative und die Thematisierung gegenhegemonialer Alternativen aber auch nicht monoperspektivisch als ,richtiger Weg' und wiederum die Alternative als alternativlose Lösung multipler Krisen präsentiert werden, denn auf diese Weise würde das Indoktrinationsproblem einer BNE nur an eine andere Stelle verlagert werden. Gelingt transformatives Lernen im Unterricht „sollte es am Ende eines Bildungsangebotes für die Lernenden möglich sein - rational und gut begründet - sogar für [...] die Weiterführung von westlich geprägten Entwicklungsdiskursen argumentieren zu können" (ebd.: 41).

Aus bildungstheoretischer Sicht bleibt allerdings der Lernbegriff zu diskutieren, welcher in der Debatte zum transformativen Lernen als Abgrenzungsfolie genutzt wird (vgl. Nohl 2017). Denn die deutschsprachige Pädagogik entwickelt bereits seit Längerem einen Lernbegriff, der weit über kumulativen Wissenserwerb hinausgeht (Göhlich \& Zirfas 2007; Göhlich et al. 2007; Mitgutsch et al. 2008; Strobel-Eisele \& Wacker 2009; Künkler 2011, 2017; Meyer-Drawe 2012; Faulstich 2014). Hier wird in der Integration solch eines pädagogischen Lernbegriffs in das Konzept des transformativen Lernens großes Potenzial für die erziehungswissenschaftliche Fundierung Transformativer Bildung gesehen. Denn wenngleich bis hierhin deren Zieldimension deutlich wurde, bleibt offen, auf welche Weise sich transformatives Lernen vollzieht. Diese theoretische Reflexion ist unmittelbar von fachdidaktischem Interesse, um Gelingensbedingungen transformativer Lernprozesse für den GW-Unterricht im Horizont einer emanzipatorischen BNE 2 auszuloten.

\section{Erfahrungstheoretische Reformulierung transformativer Lernprozesse}

Als Beitrag zur Fundierung des Konzepts Transformativer Bildung rückt hier also die Prozessualität des Lernens in den Blick und es wird dieser Fokus mit der Einsicht verschränkt, dass transformatives Lernen typischerweise mit solchen Lernerfahrungen einhergeht, die uns anrühren: "One of the most powerful tools for fostering transformative learning is providing students with learning experiences that are direct, per- 
sonally engaging and stimulate reflection upon experience" (Taylor 2007: 182). Zur Klärung des Verhältnisses von Erfahrung, Reflexion und Lernen im Zuge transformativen Lernens dient hier ein pädagogisches Verständnis von Lernen als „Umlernen“ (Buck 1989; Meyer-Drawe 1996, 2012), welches sich in der bildungsphilosophischen Tradition einer phänomenologischen Pädagogik erfahrungstheoretisch situiert. Dass in einem hermeneutisch-phänomenologischen Paradigma grundsätzlich großes Potential für die Diskussion um transformatives Lernen liegt (D'Addelfio 2017), wurde ebenso wie die Nähe zwischen transformativem Lernen und Prozessen des Umlernens (Künkler 2020) auch bereits an anderer Stelle betont. Es werden für die im Rahmen des vorliegenden Beitrags vorgenommene fachdidaktische Wendung einer pädagogischen Lerntheorie im Kontext Transformativer Bildung einige Setzungen vorgenommen, um die bildungsphilosophische Theoriearchitektur unmittelbarer anschlussfähig für das Nachdenken über nachhaltigkeitsbezogene Lernprozesse im Fachunterricht zu machen. ${ }^{5}$

Es ist in bildungsphilosophischer Hinsicht der Gedanke zentral, dass sich das Lernen als Erfahrung vollzieht und seinen Anfang darin findet, „wenn eine gewohnte Erfahrungsweise in eine Aporie gerät" (Stieve 2008: 282). Vereinfacht könnte man festhalten, dass man am Ende eines Lernprozesses um eine Erfahrung reicher ist. Lernen beschreibt dabei die Ausdifferenzierung des eigenen Erfahrungsfeldes und reicht von der Konsolidierung oder auch (graduellen) Aktualisierung eines Erfahrungshorizonts bis hin zur Eröffnung völlig neuer Erfahrungshorizonte. Das Erfahrungsfeld, mit dem wir in der Welt sind, präformiert dabei unthematisch jede Begegnung mit den Dingen (vgl. Meyer-Drawe 1996: 90). Wie wir den Dingen begegnen, was unsere Erwartungen bestätigt, was uns irritiert, verzweifeln lässt und zum Reflektieren anregt, ist maßgeblich von diesem impliziten Wissen abhängig. Dabei ist das implizite Wissen in Spuren in den Erfahrungen enthalten, sodass dieses prinzipiell thematisch und veränderbar werden kann.

Erfahrungen lassen sich dabei als Schnittstelle zwischen Welt und Selbst verstehen, indem sie das Affizierende der Welt mit dem Reflexiven des Selbst zusammenbringen: „In allen Bedeutungen von Erfahrung steckt [...] beides: eine notwendige Aktivität des Erfahrenden gegenüber den jeweiligen Phänomenen und eine unvermeidliche Passivität, in dem er es nicht in der Hand hat, was ihm wie heftig wie lange zu-

\footnotetext{
Konkret wird hier der rigorosen „Dezentralität des Subjekts“ (Künkler 2011) nicht konsequent gefolgt. Stattdessen wird im Anschluss an ein emanzipatorisches Bildungsverständnis die Rolle des Subjekts in dessen gesellschaftlicher Einbettung im Lernprozess stärker betont.
}

setzt“ (Hentig 1973: 21 f.). Zwischen diesen Polen der Erfahrung, in der Aporie, in der „das Bekannte nicht mehr gültig und das Neue noch nicht zugänglich ist" (Schratz \& Westfall-Greiter 2015: 19), liegt das transformative Potenzial des Lernens. Es gewinnt hier die bildungsphilosophische Kategorie der Erfahrung in doppelter Weise (fach)didaktische Relevanz. Zum einen lässt sich über die Erfahrung das Lernen als affektiv-sinnlicher (d.h. emotionaler) und kognitivreflexiver (d.h. rationaler) Prozess in einem verstehen, denn das Irritierende wird leiblich spürbar, benötigt aber auch die Bezugnahme, um zum Lernen zu führen. Zum anderen rüttelt die Unplanbarkeit einer Erfahrung am fachdidaktischen Verständnis inhaltliche Lernziele über die Unterrichtsgegenstände bruchlos über Unterrichtsplanung erreichen zu können. Vielmehr kommt dem gemeinsamen Einlassen auf das, was irritiert und unverstanden bleibt, große Bedeutung in pädagogischen Settings zu.

Lernprozesse sind zusätzlich qualitativ nach deren Erfahrungsintensität unterscheidbar. Es lässt sich prinzipiell eine schwache und eine starke Variante der Erfahrung voneinander abgrenzen (Waldenfels 2002: 30). Während die schwache Variante unsere Vorstellungen herauszufordern vermag oder diese auch einfach nur schlicht bestätigt, vermag es die starke Variante „uns und unsere Welt [zu] verändern“ (ebd.). Erfahrungen unterschiedlicher Intensität können zu Lernprozessen unterschiedlicher Ordnungsebenen führen. Lernprozesse zweiter Ordnung können aus einer schwachen Variante der Erfahrung resultieren und beschreiben eher die Vertiefung oder Erweiterung des Verstehens und der bisherigen Handlungsmöglichkeiten. Im Sinne eines Dazulernens innerhalb eines bestehenden Erfahrungshorizonts werden unthematische Annahmen eher verfeinert und korrigiert, d.h. die Erfahrungen „geraten im Erfahrungshorizont nur in Bewegung" (Meyer-Drawe 1996: 91). Das Dazulernen ist in diesem Sinne also kein kumulativer Prozess, sondern vollzieht sich als transformativer Prozess geringerer Intensität. Auch das Lernen zweiter Ordnung braucht die Bezugnahme zur Erfahrung, sodass diese zu einer Modifikation des Erfahrungshorizonts führen kann. Dieses Lernen beschreibt also bspw. die Verfeinerung bisherigen Wissens und Handelns oder Prozesse des gegenstandsbezogenen Hinterfragens und Reflektierens, ohne die eigenen SelbstWelt-Verhältnisse grundsätzlich in Frage zu stellen. Demgegenüber kann aus einer starken Variante der Erfahrung ein Lernprozess erster Ordnung resultieren. Dies kann dann der Fall sein, wenn unsere bisherigen Deutungsmuster und Handlungsweisen nicht mehr greifen, um in einer bestimmten irritierenden Situation eine angemessene Antwortmöglichkeit auf diese zu finden. Hiermit verbunden ist die prinzi- 
pielle Möglichkeit der Eröffnung neuer Sichtweisen auf die Gegenstände des Unterrichts, d.h. von solchen Verstehensweisen und Erfahrungshorizonten, die bislang noch kein Teil unseres Erfahrungsfeldes waren. Lernprozesse erster Ordnung besitzen damit transformativen Charakter, weil in ihnen unsere Selbst-Welt-Beziehungen mit zur Disposition stehen, unser bisher gültiges Wissen aus den Fugen gerät und wir zur grundlegenden Neuorientierung angesichts einer problematischen Situation oder Krise aufgefordert sind. Hierbei spielt die reflexive Bezugnahme auf die krisenhafte Erfahrung, d.h. die „Beobachtung der (eigenen) Beobachtung" (Schneider 2013: 6) eine große Rolle, um nicht nur zu Einsichten über die Dinge, sondern über unser Verhältnis zu den Dingen zu gelangen. Von ihrer Struktur und dem deutlichen Selbstbezug ist ein solches Umlernen also nah an Bildungsprozessen: "There are certain learning processes, which become so important for the person that his/her life-orientations get transformed. Moreover, processes of Bildung themselves always comprise of a phase of inquiry and learning" (Nohl 2017: 100). Zugleich bedeutet Lernen immer das "Lernen von etwas“ (Meyer-Drawe 2012: 187) und ist damit unmittelbar gegenstands- bzw. inhaltsbezogen und doch auch von Bildung zu unterscheiden.

Die Transformation unthematischer Erfahrungshorizonte ist kein linearer Prozess, der der Negation eines maßgeblichen Erfahrungshorizonts kausal folgt. Die Möglichkeiten des Umgangs der*des Lernenden mit den im Lernprozess spürbaren Dissonanzen spannt sich von der schlichten Erledigung, i.S. des Nicht-Einlassens auf den produktiven Fremdanspruch der einer unbekannten Situation innewohnen kann, bis zum Vollzug, i. S. des Einlassens auf Unbekanntes und Ergebnisoffenes, ohne dies vorschnell in bewährte Muster einzuordnen und damit u. U. zu glätten (vgl. Rumpf 2008: 23). Transformative Lernprozesse sind widerständig und brauchen die wiederholte Konfrontation und Herausforderung der eigenen Erfahrungshorizonte samt reflexiver Bezugnahme, um zu Veränderungen der eigenen WeltSelbst-Verhältnisse führen zu können. Im Umlernen werden alte Erfahrungen nicht gelöscht und durch neue ersetzt, sondern unsere Antwortmöglichkeiten erweitert und unser Verhältnis zur Welt und zu den Dingen verändert.

Es lassen sich somit grundsätzlich ein reflektiertes Dazulernen von einem reflexiven Umlernen unterscheiden. Während das Lernen in phänomenologischer Hinsicht grundsätzlich seinen Ausgang im Ver- sagen eines bislang gültigen Erfahrungshorizonts hat, ist dessen transformatives Potenzial von der Intensität der Erfahrung abhängig. Während das Dazulernen vom gezielten Üben bis zum gegenstandsbezogenen Hinterfragen reicht, markiert das Umlernen die Eröffnung neuer Sichtweisen. In Verbindung mit der Unterscheidung einer instrumentellen BNE 1 von einer emanzipatorischen BNE 2 lässt sich die Spezifik transformativer Lernprozesse nun gegenstandsbezogen ausdifferenzieren (siehe Tab. 1).

Tab. 1: Ebenen von Lernprozessen im Kontext einer BNE (eig. Darstellung, adaptiert nach Sterling 2011: 25)

\begin{tabular}{|c|c|c|c|c|}
\hline Lerne & Ordnungsebene & Horizonte ... & BNE-Motiv & BNE-Verständnis \\
\hline \multirow{2}{*}{$\begin{array}{l}\text { reflektiertes } \\
\text { Dazulernen }\end{array}$} & \multirow{2}{*}{$\begin{array}{c}\text { Veränderung } \\
\text { eines Verstehens- } \\
\text { und Handlungs- } \\
\text { horizonts }\end{array}$} & korrigierend & $\begin{array}{c}\text { "Dinge } \\
\text { besser tun« }\end{array}$ & \multirow{2}{*}{ instrumentell } \\
\hline & & $\begin{array}{l}\text { modifizie- } \\
\text { rend }\end{array}$ & $\begin{array}{l}\text { "Bessere } \\
\text { Dinge tun« }\end{array}$ & \\
\hline $\begin{array}{l}\text { reflexives } \\
\text { Umlernen }\end{array}$ & $\begin{array}{l}\text { Eröffnung eines } \\
\text { Verstehens- und } \\
\text { Handlungshorizonts }\end{array}$ & $\begin{array}{l}\text { transformie- } \\
\text { rend }\end{array}$ & $\begin{array}{l}\text { "Dinge an- } \\
\text { ders sehen" }\end{array}$ & zipatorisch \\
\hline
\end{tabular}

Transformative Lernprozesse, wie sie hier als bildende Erfahrungen und Prozesse des Umlernens entfaltet wurden, lassen sich didaktisch nicht einfach intendieren und im Unterricht gezielt auslösen: "We cannot teach transformation. We often cannot even identify how or why it happens. But we can teach as though the possibility always exists that a student will have a transformative experience" (Cranton 2002: 71). Damit verschiebt sich die Gestaltung von Lernumgebungen im Modus transformativen Lernens hin zur Gestaltung von Möglichkeitsräumen, in denen sich transformative Lernprozesse möglichst ereignen können. Mit dieser Akzentverschiebung stehen dann nicht die messbaren learning outcomes des Unterrichts im Fokus, sondern die zutiefst pädagogische Absicht, transformative Lernprozesse in Begegnung mit der $\mathrm{Sa}$ che und dem Austausch mit anderen möglich zu machen. Es sind diese Momente, in denen Schüler*innen „auf vielschichtige und intensive Weise in eine Auseinandersetzung mit einer Situation oder auch - für Lernprozesse besonders wichtig - mit einem Sachverhalt verwickelt" (Combe \& Gebhard 2009: 550) sind.

Gelingt die intensive Verwicklung mit der Sache - hier: der Widersprüchlichkeit und Ambivalenz nachhaltiger Entwicklung in dessen Bedeutung für Individuum und (Welt)Gesellschaft - im GW-Unterricht, können Schüler*innen im Zuge transformativer Lernprozesse Dinge anders sehen lernen, i. S. eines Lernens erster Ordnung. Das würde im besten Fall nicht weniger bedeuten, als dass sich Schüler*innen in reflexiven Umlernprozessen der eigenen Situiertheit in hegemonialen Nachhaltigkeitsdiskursen und nicht- 
nachhaltigen Strukturen reflexiv widmen können und auf diese Weise Mündigkeit in Bezug auf nachhaltigkeitsbezogene Fragestellungen, Narrative und politische Agenden erlangen und hierüber aktiv an der Gestaltung einer zukunftsfähigen Weltgesellschaft mitwirken können.

\section{Fachdidaktische Orientierungen zur Ge- staltung transformativer Lernprozesse}

Woran kann sich ein GW-Unterricht nun orientieren, der transformative Lernprozesse, wie sie hier pädagogisch präzisiert wurden, im Horizont einer emanzipatorischen BNE möglich(er) machen möchte? Mit Bezug auf die soweit zusammengetragenen Herausforderungen und theoretischen Ansprüche muss die Konzeptualisierung eines Handlungsrahmens für den GW-Unterricht fünf Dinge leisten. Der Handlungsrahmen muss: 1. Lernen auch als reflexiven Umlernprozess ermöglichen;

2. das Aufdecken und Nachspüren der diskursiven Situiertheit des eigenen Denkens und Handelns ermöglichen;

3. die Dekonstruktion eigener und alternativer Vorstellungen - u.a. von Nachhaltigkeit unter dem Primat des Wachstumsnarrativs - ermöglichen;

4. die Haltungsbildung ermöglichen und nicht auf die Durchsetzung, nachhaltiger Verhaltensweisen abzielen;

5. die ihm eingeschriebenen Logiken selbst zum Gegenstand der Reflexion werden lassen.

Zur Konzeptualisierung werden hier ein pädagogischer Lembegriff, das Prinzip Aktion \& Reflexion aus der „problemformulierenden Bildungsarbeit“ (Frei-

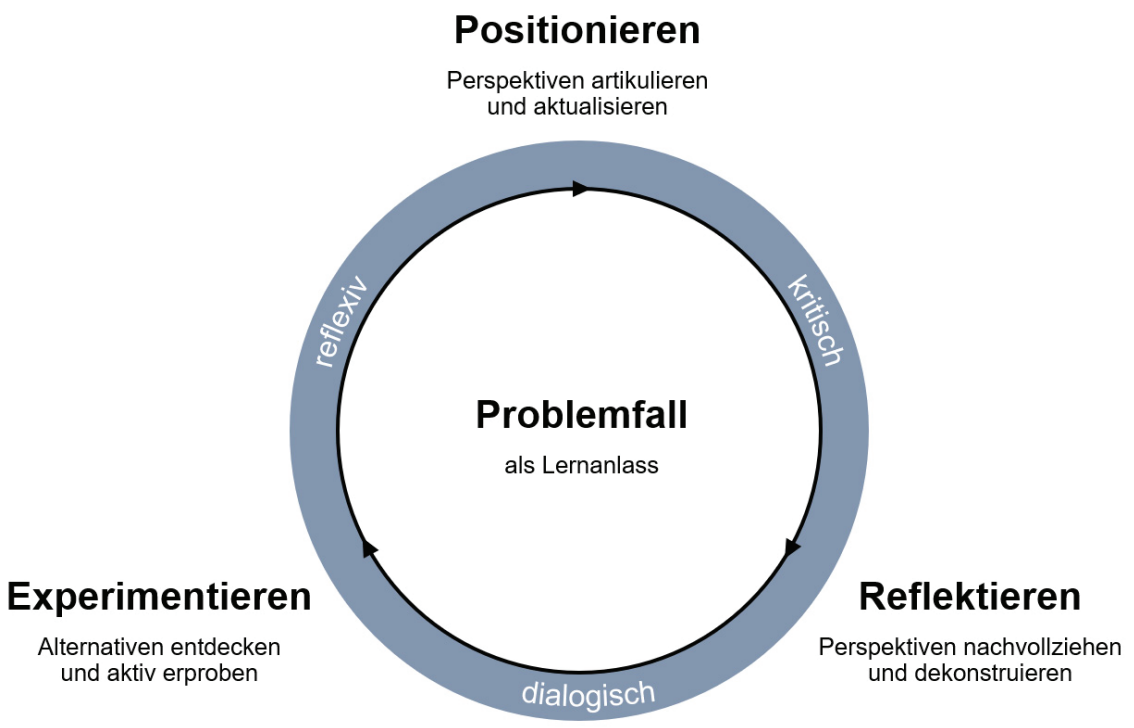

Abb. 1: Handlungsrahmen für transformatives Lernen im GW-Unterricht (eig. Darstellung) re 1973), die Schritte des „transformative learning“ (Mezirow 1997) und die didaktischen Prinzipien Konstruktion, Rekonstruktion und Dekonstruktion aus der „systemisch-konstruktivistischen Didaktik“ (Reich 2002) im Horizont einer emanzipatorischen BNE miteinander verknüpft. Der Handlungsrahmen spannt sich entlang der Unterrichtsaspekte Positionieren, Reflektieren und Experimentieren auf, kreist um einen lohnenden Problemfall und wird unterrichtspraktisch vom fachdidaktischen Prinzip der Dialogizität getragen (siehe Abb. 1).

\subsection{Ein lohnender Problemfall als Lernanlass}

Transformatives Lernen kann seinen Anfang in einem lohnenden Problemfall finden. Ein Problem wird hier dann als lohnend erachtet, wenn es einerseits lebensweltbezogen, d. h. am Erfahrungshorizont der Schüler*innen orientiert, ist und andererseits exemplarisch die dilemmatische Struktur nachhaltiger Entwicklung in sich trägt. Die Betonung der „doppelten Komplexität“ (Ohl 2013; Ohl et al. 2016; Mehren et al. 2015) - auf faktisch-fachlicher und ethisch-moralischer Ebene - stellt die Sache ins Zentrum, nicht deren unmittelbare Auflösung über vermeintlich gutes und richtiges Handeln. Die Sache ist hier die Ambivalenz und Widersprüchlichkeit einer nachhaltigen Entwicklung, die es über „die fachlichen und politischen Dimensionen der Gründe für eine nichtnachhaltige Entwicklung sachlich angemessen zu erarbeiten [gilt], um sie zu verstehen und um dadurch Perspektiven sowohl für das individuelle Handeln als auch für die Möglichkeiten kollektiven Handelns zu gewinnen" (Euler 2014: 172). Die Uneindeutigkeit der Sache birgt das Potenzial, transformative Lernprozesse anzuregen, indem diese Schüler*innen affizieren, d.h. anrühren und zum Aufmerken, Fragenstellen, Nachdenken und selbst aktiv werden anregen können (Dickel \& Schneider 2013).

\subsection{Positionieren: Perspek- tiven artikulieren und aktualisieren}

Im GW-Unterricht dient ein solcher Problemfall dazu, die Schüler*innen mit der Sache in eine produktive Begegnung zu bringen. Dabei spielt die Artikulation eigener Visionen zum Umgang mit dem erarbeiteten Dilemma eine bedeuten- 
de Rolle (Cranton 2002, 2006). In den formulierten Lösungsvorschlägen oder Handlungsempfehlungen drücken sich immer auch die unthematischen Erfahrungshorizonte der Schüler*innen aus, mit denen sie der Sache begegnen, d.h. die eigenen Vorannahmen und Bedeutungshorizonte zum Gegenstand, welche diskursiv geprägt sind. In den Artikulationen ist dieses „tacit knowledge“ (Polanyi 1985) der Schüler*innen eingeschrieben, kann also performativ erfahrbar und damit im Unterricht besprechbar werden. Der Austausch über die artikulierten Umgangsweisen im Klassenverband erlaubt die Vergewisserung über Gemeinsamkeiten und Unterschiede, d.h. das Ausloten unterschiedlicher Erfahrungshorizonte sowie deren (Un-)Vereinbarkeit und hierüber auch den Nachvollzug alternativer Sichtweisen, Visionen und Lösungsansätze. Im Gespräch lässt sich den Gemeinsamkeiten und Unterschieden nachgehen und lassen sich Gemeinsamkeiten und Widersprüche zwischen den artikulierten Perspektiven gezielt befragen.

\subsection{Reflektieren: Perspektiven nachvollziehen und dekonstruieren}

Zentral für das Gelingen transformativen Lernens ist, dass die Vorannahmen, mit denen Schüler*innen dem lohnenden Problemfall begegnet sind, thematisch werden, d.h. ins Bewusstsein rücken können. Das Bewusstwerden kann über die Reflexion einer Irritation glücken, welche sich insofern als „fruchtbarer Moment im Bildungsprozess" (Copei 1930) verstehen lässt. In diesen Momenten können bildende Erfahrungen ihren Ausgang finden und sich ihren Weg bahnen, sofern sie im Unterricht aufgegriffen und verfolgt werden (Pettig 2019). Hierzu ist die Konfrontation der zuvor über die Artikulation zugänglich gemachten Erfahrungshorizonte der Schüler*innen mit einer alternativen Umgangsweise - hier: Degrowth als gegenhegemonialem alternativen ökonomischen Leitmotiv - prinzipiell geeignet, sofern sie deren Erfahrungshorizonte übersteigt. Die Konfrontation eigener Ideen eines nachhaltigen Umgangs angesichts des Problemfalls mit alternativen Umgangsweisen und Konzepten, die bislang außerhalb des eigenen Denkens liegen, kann über die gezielte Reflexion auch zum Hinterfragen des eigenen Denkens führen: Woher kommen meine Überzeugungen und könnten diese prinzipiell auch anders sein? Inhaltlich geraten hierüber in der Nachhaltigkeitsdebatte marginalisierte und gegenhegemoniale Perspektiven in den Blick und auch die Fragen, weshalb manche Positionen in der Diskussion präsenter sind als andere, warum sich bestimmte Positionen durchsetzen können und welche Positionen (gezielt) ausgeblendet werden.

\subsection{Experimentieren: Alternativen entdecken und aktiv erproben}

Mit dem neuen Wissen lässt sich der Problemfall neu betrachten und dialektisch mögliche Auswege aus dem nachhaltigkeitsbezogenen Dilemma finden: Wenn Effizienz- und Suffizienzstrategien das Problem nur verlagern, nicht aber lösen können, könnten grundsätzliche Alternativen neue Impulse zur Problembewertung bzw. -bearbeitung liefern. Es bietet sich an, diese neuen Perspektiven auf die Sache auch zu nutzen, um deren Relevanz für das eigene Leben zu diskutieren. Hierzu zählt auch die Erarbeitung der Gründe für und wider die Gültigkeit des Entwicklungsnarrativs, indem bspw. die Bedeutung von Entwicklung für Wohlstand, Glück und Zufriedenheit zum Thema wird. Denn wenn Entwicklung und Wohlstand nicht in eins gedacht werden, könnten andere Maßstäbe herangezogen werden, um ein ,Gutes Leben' zu bestimmen, wie dies aktuell bspw. in Südamerika über das Konzept Buen Vivir, dem Recht auf Gutes Leben und die Rechte der Natur, auf Verfassungsebene diskutiert wird. Die „Reflexion der Widersprüche" (vgl. Vielhaber 1999: 17) unterschiedlicher Argumentationen und Voraussetzungen erlaubt es dann, hegemoniale, auf Entwicklung hin ausgerichtete Nachhaltigkeitsdiskurse sowie die eigene Eingebundenheit in die über diese reproduzierten strukturellen Machtverhältnisse zu erfahren und deren alternativlose Gültigkeit zu befragen. Die Aktualisierung der eigenen Positionen bedarf neben der reflexiven Bezugnahme auf das eigene Denken auch das Erproben von Alternativen, denn transformative Lernprozesse benötigen neben dem Abwägen und Formulieren von eigenen Positionen ebenso die aktiv-konstruierende Auseinandersetzung mit den diskutierten Dilemmata. Eine gelingende Transformation unthematischen Wissens führt zur Erweiterung der Artikulationsmöglichkeiten angesichts nachhaltigkeitsbezogener Fragestellungen und Dilemmata. Auf Grundlage der im Verlauf des Unterrichts thematisch gewordenen Erfahrungshorizonte, der reflexiven Ergründung der Situiertheit des eigenen Denkens und der aktiven Erkundung und Erprobung alternativer Denk- und Handlungsweisen, ist es möglich, selbstbestimmt und rational gut begründet, einen eigenen Standpunkt zu nachhaltigkeitsbezogenen Problemen und Dilemmata zu vertreten. Es bietet sich abschließend auch die Diskussion des transformativen Bildungsangebots als solches an, indem dessen jeweilige normative Implikationen und blinde Flecken mit im Unterricht thematisiert werden. 


\subsection{Dialogizität als Gestaltungsprinzip transfor- mativer Bildungsangebote}

Als zentrales unterrichtspraktisches Motiv des hier vorgeschlagenen Handlungsrahmens fungiert das Prinzip der Dialogorientierung. Über dieses wird das Bild einer klassischen Vermittlungssituation überkommen, in denen Lehrende als Expert*innen auftreten und Wissen weitergeben: „Durch Dialog hört der Lehrer der Schüler und hören die Schüler des Lehrers auf zu existieren [...]. Der Lehrer ist nicht länger bloß der, der lehrt, sondern einer, der selbst im Dialog mit den Schülern belehrt wird, die ihrerseits, während sie belehrt werden, auch lehren " (Freire 1973: 64 f.). Die Rolle der Lehrkraft ist an dieser Stelle eine zweifache: Das Prinzip der Dialogizität beruht nicht auf einem instrumentell-belehrenden Duktus, sondern auf dem gemeinsamen Einlassen auf die Dilemmata (nicht-) nachhaltiger Entwicklung und das Wertschätzen der Artikulationen der Schüler*innen ebenso, wie das gegenseitige Ergründen von Alternativen, die bislang noch nicht im Möglichkeitshorizont des Gegenübers lagen. Kurzum: Es geht in solch einem GW-Unterricht um das gemeinsame Verstehen der Sache (vgl. Dickel \& Reinhardt 2013; Dickel 2014). Die Dialogizität des Unterrichts ist vor diesem Hintergrund gerade auch für die Umsetzung des Kontroversitätsgebots und des Überwältigungsverbots von zentraler Bedeutung, wenn hierbei die „Anerkenntnis des Gegenübers" (Laub 2019: 19) und dessen reflektierte, gut begründete Position in der dialogischen Auseinandersetzung im Mittelpunkt steht und nicht dessen Überzeugung.

\section{$6 \quad$ Fazit}

Angesichts der Be- und Überlastung planetarer Grenzen, global verflochtener struktureller Ungerechtigkeit sowie systemischen Machtasymmetrien und einer ebenso kontroversen wie vielstimmigen Diskussion zu diesen und weiteren Merkmalen unserer Zeit, ist die Bedeutung mündiger Teilhabe an der demokratischen Aushandlung einer großen Transformation als Perspektive kritisch-reflexiver Bildung im GW-Unterricht überdeutlich. Damit Transformative Bildung aber im Sinne einer emanzipatorischen BNE als kritische Praxis fruchtbar werden kann, ist es im Anschluss an die Kritik an instrumentellen BNEAnsätzen unabdingbar, Bildungsprozesse nicht für die Durchsetzung einer politischen Agenda ohne realpolitische Entsprechung zu instrumentalisieren. Zugleich darf das Indoktrinationsproblem im Horizont Transformativer Bildung auch nicht einfach nur an eine andere Stelle verlagert werden. Als Zieldimension trans- formativer Bildungsangebote im GW-Unterricht ist daher festzuhalten, Schüler*innen dazu zu befähigen, an der Gestaltung einer sozial-ökologischen Transformation hin zu einer gerechten und zukunftsfähigen (Welt)Gesellschaft emanzipiert teilhaben und diese aktiv befragen und gestalten zu können.

Für die konkrete Umsetzung im Rahmen des GWUnterrichts kann der im Rahmen dieses Beitrags formulierte Handlungsrahmen ein bildungstheoretisch begründeter Ausgangspunkt sein, um transformative Lernprozesse möglich(er) zu machen. Der für die Konzeptualisierung herangezogene pädagogische Lernbegriff erwies sich dabei als äußerst ertragreich, indem transformatives Lernen als reflexive Umlernprozesse präzisiert werden konnten, deren Gestaltung mit fachdidaktischen Herausforderungen verbunden ist, die der Handlungsrahmen gezielt adressiert. $\mathrm{Ob}$ sich der hier vorgeschlagene theoretisch-konzeptionelle Handlungsrahmen im GW-Unterricht zur Gestaltung von transformativen Bildungsangeboten bewähren kann, muss an anderer Stelle diskutiert werden.

\section{Dank}

Die Veröffentlichung dieses Beitrags wurde vom Open-Access-Publikationsfonds der Universität Graz unterstützt.

\section{$7 \quad$ Literatur}

BMBF (Bundesministerium für Bildung und Frauen) (2014): Grundsatzerlass Umweltbildung für nachhaltige Entwicklung. https:/www.bmbwf.gv.at/Themen/schule/ schulrecht/rs/1997-2017/2014_20.html (06.12.2020)

BMBF (Bundesministerium für Bildung und Frauen) (2015): Unterrichtsprinzip Politische Bildung, Grundsatzerlass 2015. https://www.bmbwf.gv.at/dam/jcr:bd222bd6-faca4cf7-a0d3-9d9864d437ee/2015_12.pdf (06.12.2020)

BMWF (Bundesministerium für Wissenschaft und Forschung) (2008): Österreichische Strategie zur Bildung für nachhaltige Entwicklung. https://www.bmbwf.gv.at/ dam/jcr:682ba1b6-f2da-46ee-9627-a20c31deba0f/ bine_strategie_18299.pdf $(06.12 .2020)$

Brookfield, S. D. (2000): Transformative learning as ideology critique. In: Mezirow, J. (Hg.): Learning as transformation: Critical perspectives on a theory in progress. Jossey-Bass, San Francisco. S. 125-148.

Brookfield, S. D. (2012): Critical theory and transformative learning. In: Taylor, E. \& P. Cranton (Hrsg.): The handbook of transformative learning: Theory, research and practice. Jossey Bass, San Francisco. S. 131-146.

Budke, A. \& I. Gryl (2016): Bildung für nachhaltige Entwicklung - zwischen Utopie und Leerformel? Potentiale für die Politische Bildung im Geographieunterricht. In: Budke, A. \& M. Kuckuck (Hrsg.): Politische Bildung im Geographieunterricht. Franz Steiner, Stuttgart. S. $57-75$. 
Buck, G. (1989): Lernen und Erfahrung. WBG, Darmstadt.

Combe, A. \& U. Gebhard (2009): Irritation und Phantasie: Zur Möglichkeit von Erfahrungen in schulischen Lernprozessen. In: Zeitschrift für Erziehungswissenschaft 12(3). S. 549-557.

Copei, F. (1930): Der fruchtbare Moment im Bildungsprozess. Quelle \& Meyer, Leipzig.

Cranton, P. (2002): Teaching for transformation. In: New Directions for Adult and Continuing Education 93. S. 63-71.

Cranton, P. (2006): Fostering authentic relationships in the transformative classroom. In: New Directions for Adult and Continuing Education 109. S. 5-13.

Crutzen, P. J. \& E. Stoermer (2000): The "Anthopocene". In: Global Change Newsletter 41. S. 17-18.

Crutzen, P. J. (2002): Geology of mankind. In: Nature 415. S. 23. DOI: https://doi.org/10.1038/415023a

D’Addelfio, G. (2017): Hermeneutic Phenomenology Meets Transformative Learning. In: Laros, A., T. Fuhr \& E. W. Taylor (Hrsg.): Transformative Learning Meets Bildung. Sense Publishers, Rotterdam/Boston/Taipei. S. 127-140.

Danielzik, C.-M. (2013): Überlegenheitsdenken fällt nicht vom Himmel. Postkoloniale Perspektiven auf Globales Lernen und Bildung für nachhaltige Entwicklung. In: Zeitschrift für internationale Bildungsforschung und Entwicklungspädagogik 36(1). S. 26-33.

Dickel, M. \& A. Schneider (2013): Über Spuren. Geographie im Dialog. In: Zeitschrift für Didaktik der Gesellschaftswissenschaften 4(1). S. 56-74.

Dickel, M. \& F. Reinhardt (2013): Homo vacuus vs. Homo dialogicus - Über implizite Menschenbilder in geographischen Bildungskonzepten.In:GW-Unterricht131.S.7-15.

Dickel, M. (2014): Der Anspruch der Sache. Zur Figuration des Fragwürdigen im Dialog. In: Lange, H. \& S. Sinning (Hrsg.): Forschungs- und Lehrzusammenhang. Themenkonstitution, Ästhetik und Leiblichkeit. Schneider, Hohengehren. S. 165-186.

Euler, P. (2014): Nachhaltigkeit und Bildung: Plädoyer für ein sachhaltiges Verstehen herrschender Widersprüche. In: Umweltdachverband (Hg.): Bildung für nachhaltige Entwicklung: Jahrbuch 2014. FORUM Umweltbildung, Wien. S. 167-173.

Faulstich, P. (2014) (Hg.): Lerndebatten. Phänomenologische, pragmatische und kritische Lerntheorien in der Diskussion. Bielefeld, transcript.

Freire, P. (1973): Pädagogik der Unterdrückten: Bildung als Praxis der Freiheit. Rohwolt, Reinbek.

Getzin, S. \& M. Singer-Brodowski (2016): Transformatives Lernen in einer Degrowth-Gesellschaft. In: SOCIENCE 1(1). S. 33-46.

Göhlich, M. \& J. Zirfas (2007): Lernen. Ein pädagogischer Grundbegriff. Kohlhammer, Stuttgart.

Göhlich, M., C. Wulf \& J. Zirfas (2007): Pädagogische Theorien des Lernens. Beltz, Weinheim, Basel.
Hasse, J. (2006): Bildung für Nachhaltigkeit statt Umweltbildung? Starke Rhetorik - schwache Perspektiven. In: Hiller, B. \& M. Lange (Hrsg.): Bildung für nachhaltige Entwicklung. Perspektiven für die Umweltbildung: Vorträge und Studien. Zentrum für Umweltforschung, Münster. S. 29-43.

Hentig, H. v. (1973): Schule als Erfahrungsraum? Eine Übung im Konkretisieren einer pädagogischen Idee. Klett, Stuttgart.

Holst, J., A. Brock, M. Singer-Brodowski \& G. de Haan (2020): Monitoring Progress of Change: Implementation of Education for Sustainable Development (ESD) within Documents of the German Education System. In: Sustainability 12(10). S. 4306-25. DOI: https://doi. org/10.3390/su12104306

Kaufmann, S. (2004): Nachhaltigkeit. In: Bröckling, U. \& T. Lemke (Hrsg.): Glossar der Gegenwart. Suhrkamp, Frankfurt am Main. S. 174-181.

Kehren, Y. \& C. Winkler (2019): Nachhaltigkeit als Bildungsprozess und Bildungsauftrag. In: Leal Filho, W. (Hg.): Aktuelle Ansätze zur Umsetzung der UN-Nachhaltigkeitsziele. Springer, Wiesbaden. S. 373-391.

Koller, H.-C. (2017): Bildung as a Transformative Process. In: Laros, A., T. Fuhr \& E. W. Taylor (Hrsg.): Transformative Learning Meets Bildung. Sense Publishers, Rotterdam/Boston/Taipei. S. 33-42. DOI: https://doi. org/10.1007/978-94-6300-797-9_3

Künkler, T. (2011): Lernen in Beziehung: Zum Verhältnis von Subjektivität und Relationalität in Lernprozessen. transcript, Bielefeld.

Künkler, T. (2017): Lernen in Beziehungen. Entwurf einer relationalen Theorie des Lernens. In: Jugendhilfe 55(5). S. 458-463.

Künkler, T. (2020): Von starken Wertungen, Umwertungen und der Fülle des Lebens. Charles Taylor lesen als Umlernprozess. In: Balzer, N., J. Beljan \& J. Drerup (Hrsg.): Charles Taylor - Perspektiven der Erziehungsund Bildungsphilosophie. Mentis, Paderborn. S. 41-58.

Laub, J. (2019): Haltung im Geographieunterricht? Zur Bedeutung eines pädagogischen Begriffs für die Unterrichtspraxis im Fach Geographie. In: OpenSpaces (2)1. S. 9-22.

Lehner, M. \& I. Gryl (2019): Neoliberalismus in NRWs Sachunterrichtsbüchern? In: GW-Unterricht 156. S. 5-18.

Lingenfelder, J. (2020): Was bedeutet Transformative Bildung im Kontext sozial-ökologischer Krisen? In: Außerschulische Bildung 51(1). S. 52-56.

Marchand, S. (2015): Nachhaltig entscheiden lernen. Urteilskompetenzen für nachhaltigen Konsum bei Jugendlichen. Klinkhardt, Bad Heilbrunn.

Mehren, M., R. Mehren, U. Ohl \& C. Resenberger (2015): Die doppelte Komplexität geographischer Themen - eine lohnenswerte Herausforderung für Schüler und Lehrer. In: Geographie aktuell und Schule 216. S. 4-11.

Meyer-Drawe, K. (1996): Vom anderen lernen: Phänomenologische Betrachtungen in der Pädagogik. In: Borelli, 
M. \& J. Ruhloff (Hrsg.): Deutsche Gegenwartspädagogik Band II. Schneider Hohengehren, Baltmannsweiler. S. 85-98.

Meyer-Drawe, K. (2012): Diskurse des Lernens. Wilhelm Fink, München.

Mezirow, J. (1997): Transformative Erwachsenenbildung. Schneider Hohengehren, Baltmannsweiler.

Mitgutsch, K., E. Sattler, K. Westphal \& I. M. Breinbauer (Hrsg.) (2008): Dem Lernen auf der Spur. Die Pädagogische Perspektive. Klett-Cotta, Stuttgart.

Nohl, A.-M. (2017): Problematic Commonalities of Bildung and Transformative Learning. In: Laros, A., T. Fuhr \& E. W. Taylor (Hrsg.): Transformative Learning Meets Bildung. Sense Publishers, Rotterdam/Boston/Taipei: Sense Publishers. S. 97-106.

Ohl, U. (2013): Komplexität und Kontroversität: Herausforderungen des Geographieunterrichts mit hohem Bildungswert. In: Praxis Geographie 43(3). S. 4-8.

Ohl, U., C. Resenberger \& T. Schmitt (2016): Zur politischen Dimension der Frage nach "gutem" Handeln im Geographieunterricht. In: Budke, A. \& M. Kuckuck (Hrsg.): Politische Bildung im Geographieunterricht. Franz Steiner, Stuttgart. S. 89-96.

O'Sullivan, E. (1999): Transformative Learning: Educational Vision for the $21^{\text {st }}$ Century. Palgrave, New York.

O'Sullivan, E. (2002): The project and vision of transformative education: Integral transformative learning. In: O'Sullivan, E., A. Morrell \& M. A. O’Connor (Hrsg.): Expanding the boundaries of transformative learning. Palgrave, New York. S. 1-12.

O'Sullivan, E. (2012): Deep transformation: Forging a planetary worldview. In: Taylor, E. W. \& P. Cranton (Eds.): The handbook of transformative learning: Theory, research and practice. Jossey-Bass, San Francisco. S. 162-177.

Pettig, F. (2019): Kartographische Streifzüge: Ein Baustein zur phänomenologischen Grundlegung der Geographiedidaktik. transcript, Bielefeld.

Polanyi, M. (1985): Implizites Wissen. Suhrkamp, Frankfurt am Main.

Reich, K. (2002): Systemisch-konstruktivistische Didaktik. Eine allgemeine Zielbestimmung. In: Voß, R. (Hg.): Die Schule neu erfinden: systemisch-konstruktivistische Annäherungen an Schule und Pädagogik. Luchterhand, Neuwied/Kriftel. S. 70-91.

Rumpf, H. (2008): Lernen als Vollzug und als Erledigung. In: Mitgutsch, K., E. Sattler, K. Westphal \& I. M. Breinbauer (Hrsg.): Dem Lernen auf der Spur. Klett-Cotta, Stuttgart. S. 21-32.

Scharf, C. \& I. Gryl (2020): Innovativität - Gerechtigkeit - Nachhaltigkeit. Eine Erweiterung des Konzepts Innovativität um die Theorie der Gerechtigkeit und dessen Anwendung im Kontext bon Bildung für nachhaltige Entwicklung. In: GW-Unterricht 159. S. 16-30. DOI: https://doi.org/10.1553/gw-unterricht159s16

Schild, K., M. Leng \& T. Hammer (2019): Die Rolle von Transformativem Lernen für eine Bildung für Nachhal- tige Entwicklung an der Hochschule. In: Bulletin VSHAEU 45(2). S. 34-40. DOI: https://doi.org/10.7892/ boris. 132869

Schneider, A. (2013): Geographiedidaktische Reflexivität. LIT, Berlin, Münster.

Schneidewind, U. (2019): Die große Transformation. Eine Einführung in die Kunst gesellschaftlichen Wandels. Fischer, Frankfurt am Main.

Schratz, M. \& T. Westfall-Greiter (2015): Lernen als Erfahrung: Ein pädagogischer Blick auf Phänomene des Lernens. In: Dumont, H., D. Istance \& F. Benavides (Hrsg.): The Nature of Learning - Die Natur des Lernens. Beltz, Weinheim/Basel. S. 14-33.

Schuler, S. \& D. Kanwischer (2013): Bildung für nachhaltige Entwicklung: Globales Lernen und Umweltbildung im Geographieunterricht. In: Kanwischer, D. (Hg.): Geographiedidaktik. Ein Arbeitsbuch zur Gestaltung des Geographieunterrichts. Borntraeger, Stuttgart. S. 164-175.

Selby, D. \& F. Kagawa (2010). Runaway Climate Change as Challenge to the 'Closing Circle' of Education for Sustainabale Development. In: Journal of Education for Sustainable Development 4(1). S. 37-50.

Singer-Brodowski, M. (2016): Transformative Bildung durch transformatives Lernen. Zur Notwendigkeit der erziehungswissenschaftlichen Fundierung einer neuen Idee. In: Zeitschrift für internationale Bildungsforschung und Entwicklungspädagogik 39(1). S. 13-17.

Steffen, W., K. Richardson, J. Rockström, S. E. Cornell, I. Fetzer, E. M. Bennett, R. Biggs, S. R. Carpenter, W. de Vries, C. A. de Wit, C. Folke, D. Gerten, J. Heinke, G. M. Mace, L. M. Persson, V. Ramanathan, B. Reyers \& S. Sörlin (2015): Planetary boundaries: Gioding human development on a changing planet. In: Science 347(6223). S. 736-745. DOI: https://doi.org/10.1126/ science. 1259855

Sterling, S. (2011): Transformative Learning and Sustainability: Sketching the Conceptual Ground. In: Learning and Higher Education 5. S. 17-33.

Stieve, C. (2008): Von den Dingen lernen. Die Gegenstände unserer Kindheit. Wilhelm Fink, München.

Strobel-Eisele, G. \& A. Wacker (Hrsg.) (2009): Konzepte des Lernens in der Erziehungswissenschaft: Phänomene, Reflexionen, Konstruktionen. Klinkhardt, Bad Heilbrunn.

Taylor, E. (2007): An update of transformative learning theory: a critical review of the empirical research (19992005). In: International Journal of Lifelong Education (26)2. S. 173-191.

UN (2015): Transforming our World: The 2030 Agenda for Sustainable Development. http://www.un.org/ga/ search/view_doc.asp?symbol=A/RES/70/1\&Lang=E $(06.12 .2020)$

UNESCO (2005): United Nations Decade of Education for Sustainable Development (2005-2014): International Implementation Scheme. UNESCO, Paris.

UNESCO (2020): Education for Sustainable Development. A roadmap. UNESCO, Paris. 
Vare, P. \& W. Scott (2007): Learning for a change: Exploring the Relationship Between Education and Sustainable Development. In: Journal of Education for Sustainable Development 1(2). S. 191-198.

Vielhaber, C. (1999): Vermittlung und Interesse - Zwei Schlüsselkategorien fachdidaktischer Grundlegungen im Geographie- und Wirtschaftskundeunterricht. In: Vielhaber, C. (Hg.): Fachdidaktik kreuz und quer: Vom Vermittlungsinteresse bis zum Methodenstreit - Von der Spurensuche bis zum Raumverzicht. Institut f. Geographie und Regionalforschung, Wien. S. 9-26.

Waldenfels, B. (2002): Bruchlinien der Erfahrung. Suhrkamp, Frankfurt am Main.
Wals, A. E. J., F. Geerling-Eijf, F. Hubeek, S. van der Kroon \& J. Vader (2008): All Mixed Up? Instrumental and Emancipatory Learning Toward a More Sustainable World: Considerations for EE Policymakers. In: Applied Environmental Education and Communication (8)3. S. 5565. DOI: https://doi.org/10.1080/15330150802473027

WBGU (Wissenschaftlicher Beirat der Bundesregierung Globale Umweltveränderungen) (2011): Welt im Wandel: Gesellschaftsvertrag für eine Große Transformation. WBGU, Berlin.

Wehling, H.-G. (1977): Konsens à la Beutelsbach? Nachlese zu einem Expertengespräch. In: Schiele, S. \& H. Schneider (Hrsg.): Das Konsensproblem in der politischen Bildung. Ernst Klett, Stuttgart. S. 173-184. 